\title{
An Optimal Life Insurance Policy in the Continuous-Time Investment-Consumption Problem
}

\author{
Hideki Iwaki ${ }^{1}$, Yusuke Osaki ${ }^{2}$ \\ ${ }^{1}$ Faculty of Business Administration, Kyoto Sangyo University, Kyoto, Japan \\ ${ }^{2}$ Faculty of Economics, Osaka Sangyo University, Osaka, Japan \\ Email: iwaki@cc.kyoto-su.ac.jp,osaki@eco.osaka-sandai.ac.jp
}

Received December 8, 2012; revised February 11, 2013; accepted February 18, 2013

Copyright (C) 2013 Hideki Iwaki, Yusuke Osaki. This is an open access article distributed under the Creative Commons Attribution License, which permits unrestricted use, distribution, and reproduction in any medium, provided the original work is properly cited.

\begin{abstract}
This paper considers an optimal life insurance for a household subject to mortality risk. The household receives wage income continuously, which could be terminated by unexpected premature loss of earning power. In order to hedge the risk of losing income stream, the household enters a life insurance contract. The household may also invest their wealth into a financial market. Therefore, the problem is to determine an optimal insurance/investment/consumption strategy. To reflect a real-life situation better, we consider an incomplete market where the household cannot trade insurance contracts continuously. We provide explicit solutions in a fairly general setup.
\end{abstract}

Keywords: Life Insurance; Investment/Consumption Model; Martingale; Convex Duality

\section{Introduction}

We consider a household whose income stream relies on one particular member of the family. The household has an incentive to buy a life insurance contract to mitigate mortality risk of the wage earner. The investment time horizon of the household is $[0, T]$ where $T$ denotes the planned retirement time of that person. That is, the household expects to receive wage income at rate $y(t)$ continuously until time $T$, which could be terminated before time $T$ by some unexpected loss of earning power (e.g. death). Accordingly, it is natural to assume that the household buys an insurance that terminates at time $T$. In other words, the insurance coverage is effective until and upon time $T$. The mortality risk is modeled by a first arrival of a certain Poisson process $N=\{N(t) ; t \geq 0\}$ with intensity process $\lambda=\{\lambda(t) ; t \geq 0\}$. We denote the random time of that event by $\tau$. The household buys $n$ shares of an insurance policy by paying a lump-sum premium of $n \times p_{0}$ at time 0 . We assume the premium per share, $\quad p_{0}$ is determined exogenously and $n$ is one of the decision variables. The insurance company pays insurance amount $X$ per share that depends on the time of Poisson arrival $\tau$. Therefore, if the household purchases $n$ shares, the payment at $\tau \leq T$ is $n \times X(\tau)$.

Given the initial endowment at time 0 , the household decides on the number of insurance contracts $n$ and in- vests the rest of the money available into the financial market. In the case of $\tau \leq T$, the household receives insurance money $n X(\tau)$ and shall use the money for consumption and/or additional investment in the financial market. On the other hand, if $\tau>T$, the insurance contract terminates and the insured person retires at $T$. The household tries to maximize its utility for the entire time horizon $[0, T]$. See the next section for complete mathematical formulation. It should be emphasized here that the decision maker in our problem is the "household" (i.e. the whole family), not the insured person. Hence after time $\tau$, consumption still continues.

Although the financial market (excluding insurance contracts) is assumed to be complete, the household's inability to trade insurance contracts makes the whole model incomplete. We show, by using the convex duality method, that if a certain $n$ solves the dual problem, that $n$ also solves the original utility maximization problem. We then explicitly compute the corresponding consumption and wealth processes. However, we need to specify a state price density process with respect to the mortality risk in order to solve the dual problem, and it is generally quite difficult to derive the process explicitly. To avoid this difficult issue, alternatively, we provide such a condition that the state price density process is given as a constant 1 . In other words, we provide a condition such that the household evaluates the insurance benefit as its 
expected value of discounted cash-flow under the physical probability measure. Under the condition, provided that insurance benefit is a linear function of $n$, the household shall invest all the initial endowment either in the insurance contract or in the financial market, depending on the relationship between the insurance premium and the expected discount value of insurance benefit. This is natural because the financial market excluding insurance contracts is complete, if these two quantities are not equal, the household takes a full advantage of possible mispricing in the insurance contract. On the other hand, if these quantities are equal, the household does not have a clue as to how it should determine the optimal number of insurance contracts. Finally, we provide the optimal portfolio strategy for each $n$. Hence this paper analyzes the household's optimal behavior when the household faces the insurance contract and the financial market.

\section{Literature Review}

We briefly discuss this paper's position in the existing literature. While this paper considers insurance payment (at $\tau$ ) as a source of income to the bereft family members for the rest of the time horizon, previous treatments of insurance in the literature are in essence from insurers' point of view. The main purpose is to calculate insurance premium of various contracts whose payment is exogenously given. For example, [1,2] examined option-like features contained in the insurance contacts. The fair premium of an equity-linked life insurance contract is calculated in [3,4], while [5] calculated the reserves in a stochastic mortality and interest rate model. See [6]. See also $[7,8]$ for determining insurance premium in a multiperiod economy and in a continuous-time economy, respectively.

In contrast, this paper discusses an optimal insurance purchase from the standpoint of households. The problem treated in this paper can be seen as an extension of the security allocation problem originally studied by [9, 10]. In his model, only a riskless security and a risky security are considered and the problem is to obtain an optimal portfolio rule so as to maximize the expected utility from consumption. Since then, the model has been extended to various directions. [11] included life insurance decisions in the Merton model. He assumed a specific diffusion for the risky asset and a complete market where the investor can trade life insurance contracts continuously. The investor in [11] maximizes the utility until uncertain time of death. [12] used a discrete-time model to derive the demand function for life insurance. [13] extended to a multi-period model but did not include risky assets in the asset portfolio. [14], in one-period model, performed a comprehensive study of the insurance-investment-consumption problem and analyzed effects of parameters on individuals' insurance purchase, consumption, and stock investment decisions by using two different individual groups: one with exponential utility and the other with power utility. Also, [15] studied a life time model in which a "human capital" is considered, as in [11], to represent the present value of the total wage income to be obtained in the future. By including the human capital in their security allocation model, they succeeded in explaining the relationship between the age of an economic agent and his/her optimal investment strategy. See also [16-18] as examples of such extensions.

Our current article contrasts with these papers in that we use a continuous-time framework with general utility functions and general underlying diffusions. Moreover, in order to make the model more realistic, we assume that the household cannot trade insurance contracts (unlike [11]) and incorporate the fact that the bereft family would use the money from the insurance contract to continue their consumption until the fixed time $T$. It should be noted that [19] showed the existence of solutions for the investment-consumption problem with a random endowment in a general semimartingale model. Our current paper, though, has distinct merits in the sense that we obtain an explicit solution for a fairly general utility function and hence make economic implications much clearer. Moreover, while the random endowment in [19] is given exogenously, our random endowment (i.e., insurance money) here can be controlled by changing the number of shares of the insurance contract.

This paper is organized as follows. In the next section, we formulate our problem in a rigorous manner. We solve the problem in the following section and discuss possible extensions after we present our main results. We defer the detailed proofs to Appendix. Throughout this paper, all the random variables considered are bounded almost surely (a.s.) to avoid unnecessary technical difficulties. Equalities and inequalities for random variables hold in the sense of almost surely.

\section{The Model}

Let us consider a complete filtered probability space $\left(\Omega, \mathcal{F},\left(\mathcal{F}_{t}\right)_{t \in \mathbb{R}_{+}}, \mathbb{P}\right)$ that hosts a Brownian motion $B:=\{B(t) ; t \geq 0, B(0)=0\}$ and a Poisson Process $N:=\{N(t) ; t>0, N(0)=0\}$ with the intensity process $\lambda:=\{\lambda(t) ; t \geq 0\}$. Let $\mathcal{F}_{t}^{B}:=\sigma\{B(s) ; s \leq t\}, t \in[0, T]$.

We denote the $\mathbb{P}$-augmentation of filtration by $\mathbb{F}^{B}:=\left\{\mathcal{F}_{t}^{B} ; t \in(0, T)\right\}$. The Brownian motion is the source of randomness other than the time $\tau$ :

$$
\tau:=\inf \{t>0 ; N(t)=1\},
$$


which denotes the time of the insured person's loss of earning power (e.g. death). We assume that the Poisson process $N$ and the Brownian motion $B$ are mutually independent. Let $\mathcal{F}_{t}^{N}:=\sigma\left\{1_{\{\tau \leq s\}} ; s \leq t\right\}$ where $1_{E}$ denotes the indicator function of event $E \in \mathcal{F}$ meaning that $1_{E}=1$ if $E$ is true and $1_{E}=0$ otherwise. The $\mathbb{P}$ augmentation of the filtration is denoted by

$\mathbb{F}^{N}:=\left\{\mathcal{F}_{t}^{N} ; t \in[0, T]\right\}$. Clearly, $\tau$ is an $\mathbb{F}^{N}$-stopping time, but not an $\mathbb{F}^{B}$-stopping time. Now, let

$\mathcal{F}_{t}:=\mathcal{F}_{t}^{B} \vee \mathcal{F}_{t}^{N}, t \in[0, T]$ and its $\mathbb{P}$-augmentations $\mathbb{F}:=\left\{\mathcal{F}_{t} ; t \in[0, T]\right\}$. It is assumed that $\mathbb{F}$ satisfies the usual conditions regarding right-continuity and completeness. The conditional expectation operator given $\mathcal{F}_{t}$ is denoted by $\mathbb{E}_{t}$ with $\mathbb{E}=\mathbb{E}_{0}$.

Suppose that the current time is 0 , and let $T>0$ be the termination time of an insurance contract which is set to be the same as the retirement time. We consider a continuous-time economy in $[0, T]$ that consists of the insurance contract and a financial market. The financial market is assumed to be frictionless and perfectly competitive ${ }^{1}$.

The household may receive cash flow from various sources of income. But for simplicity, we assume that it relies on one member's income stream:

$y=\{y(t) ; t \in[0, T]\} \quad$ (called income process hereafter) which is given exogenously until time $T$. To hedge the risk of loss of income flow at time $\tau<T$, the household buys an insurance policy described as follows: Once the household buys $n$ shares of the policy by paying the insurance premium amounts $p_{0} \times n$ at time 0 , the insurance company makes an insurance payment in the amount of

$$
n \times X(t)=n \times(1+H(t))
$$

at time $t=\tau \wedge T$. Here $H:[0, T] \mapsto \mathbb{R}_{+}$is given exogenously, representing payment schedule until time $T$. In case $\tau>T$, the policy pays 1 dollar per share at time $T$. In order to avoid unnecessary complications, we assume that the schedule function satisfies the following assumption.

Assumption $1 \quad H:[0, T] \mapsto \mathbb{R}_{+}$is a nonincreasing continuous function with $H(T)=0$.

Here we note that $H(T)=0$ means that the insurance amount when $\tau$ occurs at time $T$ and the guaranteed insurance amount (which is unity) on the set $\{\tau(\omega)>T\}$ coincide.

Let $c=\{c(t) ; t \in[0, T]\}$ be the consumption process to be determined by the household. It is assumed that income and consumption processes are adapted to $\mathbb{F}$. In

\footnotetext{
${ }^{1}$ A financial market is said to be frictionless if the market has no transaction costs, no taxes, and no restrictions on short sales (such as margin requirements), and asset shares are divisible, while it is called perfectly competitive if each agent believes that he/she can buy and sell as many assets as desired without changing the market price.
}

the financial market, there is a riskless security whose time $t$ price is denoted by $S_{0}(t)$. The riskless security evolves according to the differential equation;

$$
\frac{\mathrm{d} S_{0}(t)}{S_{0}(t)}=r(t) \mathrm{d} t, t \in[0, T],
$$

where $r(t)$ is a positive, predictable process with respect to $\mathbb{F}^{B}$. The household can also invest their wealth into a risky security whose time $t$ price is denoted by $S_{1}(t)$. The risky security evolves according to the stochastic differential equation (abbreviated SDE);

$$
\frac{\mathrm{d} S_{1}(t)}{S_{1}(t)}=\mu(t) \mathrm{d} t+\sigma(t) \mathrm{d} B(t), t \in[0, T],
$$

where $\mu(t)$ and $\sigma(t)$ are progressively measurable processes with respect to $\mathbb{F}^{B}$.

Let $\pi(t)$ be the amount to be invested into the risky security at time $t$. The process $\pi=\{\pi(t) ; t \in[0, T]\}$ is referred to as a portfolio process. Now, given a portfolio process $\pi$, a consumption process $c$, the number of shares of the insurance policy $n$ and an income process $y$, the wealth process $W=\{W(t) ; t \in[0, T]\}$ is defined by

$$
W(t):=\left\{\begin{array}{l}
W_{0}-n p_{0}+\int_{0}^{t}(r(s) W(s)+y(s)-c(s)) \mathrm{d} s \\
\quad+\int_{0}^{t} \pi(s)[(\mu(s)-r(s)) \mathrm{d} s+\sigma(s) \mathrm{d} B(s)] \\
\quad \text { if } t \in[0, \tau \wedge T), \\
W(\tau-)+n X(\tau)+\int_{\tau}^{t}(r(s) W(s)-c(s)) \mathrm{d} s \\
+\int_{\tau}^{t} \pi(s)[(\mu(s)-r(s)) \mathrm{d} s+\sigma(s) \mathrm{d} B(s)] \\
\text { if } t \in[\tau \wedge T, T],
\end{array}\right.
$$

where $W_{0}$ is a given initial wealth which is assumed to be a positive constant.

In this paper, we assume that, given the intensity process $\lambda$, the conditional survival probability of $\tau$ is given by

$$
\mathbb{P}\{\tau>t \mid \lambda\}=\exp \left\{-\int_{0}^{t} \lambda(u) \mathrm{d} u\right\}, t \in[0, T] .
$$

That is, the intensity process $\lambda$ plays the role of the hazard rate,

$$
\lambda(t)=\lim _{\Delta \downarrow 0} \frac{1}{\Delta} \mathbb{P}\left\{t<\tau \leq t+\Delta \mid \tau>t, \mathcal{F}_{t}^{B}\right\} .
$$

A Poisson process $N$ driven by that (stochastic) intensity process is called a Cox process, which is also known as a doubly stochastic Poisson process. See, for example, [20] for details. In this case, we have $\mathbb{P}\left\{\tau>t \mid \mathcal{F}_{t}^{B}\right\}=\mathbb{P}\left\{\tau>t \mid \mathcal{F}_{T}^{B}\right\}$ for $t \leq T$. Note that, in this setting, the infinitesimal increments $d B(t)$ and $d N(t)$ are conditionally independent given $\mathcal{F}_{t}^{B}$. Also, 
the process $M_{\lambda}=\left\{M_{\lambda}(t), t \in[0, T]\right\}$ defined by

$$
M_{\lambda}(t):=\int_{0}^{t} 1_{\{N(s-)=0\}}[\mathrm{d} N(s)-\lambda(s) \mathrm{d} s]
$$

is an $\mathbb{F}$-martingale (i.e. the integral $\int_{0}^{t} 1_{\{N(s-)=0\}} \lambda(s) \mathrm{d} s$ is the $\mathbb{F}$-compensator (see [21])).

Definition $1 \mathrm{~A}$ consumption and wealth pair $(c, W)$ is called feasible if $c(t) \geq 0, W(t)>-\infty$ for $t \in[0, T]$, $W(T) \geq 0$ and it satisfies (3). We denote a class of feasible pairs $(c, W)$ by $\mathcal{C}$.

Recall that the household consumes the wage income and, if any, insurance money to maximize the expected discounted utility from consumption $c$ and terminal wealth $W(T)$. Let $U_{1}:(0, \infty) \rightarrow \mathbb{R}$ be the utility function of the household from consumption, and let $U_{2}:(0, \infty) \rightarrow \mathbb{R}$ be the utility function of the household from the terminal wealth. In order to guarantee the existence of a unique optimal solution as an internal point, we assume that the following assumption holds in the sequel (See the proof of Proposition 1 and that of Proposition 2 in Appendix).

Assumption $2 \mathrm{We}$ assume that our utility functions satisfy the following:

1) $U_{i} \quad(i=1,2)$ are strictly increasing, strictly concave and twice continuously differentiable with properties

$$
\begin{aligned}
U_{i}^{\prime}(\infty):=\lim _{x \rightarrow \infty} U_{i}^{\prime}(x)=0, & \text { of consumption process, portfolio proc } \\
U_{i}^{\prime}(0+):=\lim _{x \downarrow 0} U_{i}^{\prime}(x)=\infty, \quad i=1,2 . & \text { ber of shares of the insurance policy } \\
& \max \mathbb{E}\left[\int_{0}^{\tau \wedge T} \exp \left\{-\int_{0}^{t} \rho(s) \mathrm{d} s\right\} U_{1}(c(t)) \mathrm{d} t+\exp \left\{-\int_{0}^{\tau \wedge T} \rho(s) \mathrm{d} s\right\} V(W(\tau \wedge T))\right]
\end{aligned}
$$

with

$$
V(W(\tau \wedge T)):=\max \mathbb{E}\left[\int_{\tau \wedge T}^{T} \exp \left\{-\int_{\tau \wedge T}^{t} \rho(s) \mathrm{d} s\right\} U_{1}(c(t)) \mathrm{d} t+\exp \left\{-\int_{\tau \wedge T}^{T} \rho(s) \mathrm{d} s\right\} U_{2}(W(T)) \mid \mathcal{F}_{\tau \wedge T}\right]
$$

where the maximum is taken over by the feasible consumption and wealth pairs, $(c, W) \in \mathcal{C}$ under the budget constraint Equation (3).

In the next section, we shall solve the problem (MP) by applying the martingale approach in an incomplete market (see [18] and [22]).

\section{Main Results}

In order to apply the martingale approach, we need to specify a state price density process first. Let $\mathcal{P}$ be a class of positive and predictable stochastic processes;

$$
\mathcal{P}=\left\{\psi(t) ; \int_{0}^{\tau \wedge T} \psi(t) \mathrm{d} t<\infty, t \in[0, \tau \wedge T]\right\} .
$$

For each $\psi=\{\psi(t) ; t \in[0, \tau \wedge T]\} \in \mathcal{P}$, the state price density process is given by
2) For any $c \in(0, \infty)$ there exist real numbers $a \in(0, \infty)$ and $b \in(0, \infty)$ satisfying $a U_{i}^{\prime} \geq U_{i}^{\prime}(b c)$.

Also, in order to represent time-preference of the household, we introduce a time-discount factor

$$
\exp \left\{-\int_{0}^{t} \rho(s) \mathrm{d} s\right\}, \quad t \in[0, T],
$$

where the process $\rho=\{\rho(t), t \in[0, T]\}$ is adapted to $\mathbb{F}$. A natural problem for the household is as follows: Given the initial wealth $W_{0}$, the household decides how many insurance contracts to buy at time zero to protect from the risk of the Poisson event. The rest of the money $\tau \leq T$, the household receives the insurance money $n X(\tau)$ as in Equation (1) and re-solves the optimal investment-consumption problem Equation (7) by using the sum of the wealth at $\tau, W(\tau-)$ and the insurance and, if $\tau>T$, the problem reduces to an ordinary decides on the number of insurance contract $n$ at time zero along with the optimal consumption-investment pair to maximize the overall utility. Mathematically, it is tated as follows:

(MP) Given the discount process $\rho$ and utility functions $U_{i}(x), i=1,2$, find an optimal triplets consisting consumption process, portfolio process and the number of shares of the insurance policy $(\hat{c}, \hat{w}, \hat{n})$ to solve $W_{0}-n p_{0}$ can be invested in the financial market. If

$$
\chi(t):=\beta(t) \chi^{B}(t) \chi^{N}(t)
$$

where

$$
\begin{aligned}
\beta(t) & :=\exp \left\{-\int_{0}^{t} r(s) \mathrm{d} s\right\}, \\
\chi^{N}(t):= & \left(\frac{\psi(\tau)}{\lambda(\tau)} 1_{\{\tau \leq t\}}+1_{\{\tau>t\}}\right) \\
& \times \exp \left\{\int_{0}^{t \wedge \tau}(\lambda(s)-\psi(s)) \mathrm{d} s\right\},
\end{aligned}
$$

and

$$
\chi^{B}(t):=\exp \left\{-\int_{0}^{t} \xi(s) \mathrm{d} B(s)-\frac{1}{2} \int_{0}^{t} \xi^{2}(s) \mathrm{d} s\right\},
$$

with 


$$
\xi(t):=\frac{\mu(t)-r(t)}{\sigma(t)}, t \in[0, T] .
$$

Note that Equations (8) and (10) say that the state price density process $\chi$ is determined once the intensity process $\psi$ is specified. Here and hereafter, we denote the conditional expectation operator given $\mathcal{F}_{t}$ under the equivalent martingale measure $\mathbb{Q}$ by $\mathbb{E}_{t}^{\mathbb{Q}}$ with $\mathbb{E}^{\mathbb{Q}}=\mathbb{E}_{0}^{\mathbb{Q}}$.

Remark 1 The following facts are well known. See for example [21] and [23].

1) The state price density process $\chi=\{\chi(t) ; t \in[0, T]\}$ is such a process that $\chi(0)=1,0<\chi(t)<\infty$, and for each $t \in[0, T]$ and for any $s>t, s \in[0, T]$,

$$
\mathbb{E}_{t}\left[\chi(s) S_{j}(s)\right]=\chi(t) S_{j}(t), j=0,1,
$$

i.e. each process $\left\{\chi(t) S_{j}(t), t \in[0, T]\right\}, j=0,1$, is martingale under $\mathbb{P}$.

2) The equivalent martingale measure $\mathbb{Q}$ is given by

$$
\frac{\mathrm{d} \mathbb{Q}}{\mathrm{d} \mathbb{P}}=\frac{\chi(T)}{\beta(T)},
$$

3) The process $\psi$ represents the intensity process under the equivalent martingale measure $\mathbb{Q}$.

We solve the problem (MP) in two steps. First, for a given $n \in \mathbb{R}_{+}$, we solve the problem by applying the martingale methods for optimal portfolio selection problems in incomplete market. Second, we derive the value of $n$ which maximizes the value function that is derived in the first step.

In the following, in order to make the dependence on $\psi \in \mathcal{P}$ explicit, we denote the state price density by $\chi_{\psi}(t)=\beta(t) \chi^{B}(t) \chi_{\psi}^{N}(t), t \in[0, T]$, where

$$
\begin{aligned}
\chi_{\psi}^{N}(t)= & \left(\frac{\psi(\tau)}{\lambda(\tau)} 1_{\{\tau \leq t\}}+1_{\{\tau>t\}}\right) \\
& \times \exp \left\{-\int_{0}^{t \wedge \tau}(\psi(s)-\lambda(s)) \mathrm{d} s\right\},
\end{aligned}
$$

See also Equation (10). Similarly, $\mathbb{Q}_{\psi}$ denotes the equivalent martingale measure associated with the state price density $\chi_{\psi}$, which is given by

$$
\mathrm{d} \mathbb{Q}_{\psi} / \mathrm{d} \mathbb{P}=\chi_{\psi}(T) / \beta(T) .
$$

For a given consumption and wealth pair $(c, W)$, the next result provides a necessary condition regarding its feasibility in the market.

Lemma 1 If a consumption and wealth pair $(c, W)$ is in $\mathcal{C}$ (as in Definition 1), then it satisfies the following inequalities.

$$
\left\{\begin{array}{l}
\mathbb{E}^{\mathbb{Q}_{\psi}}\left[\int_{0}^{\tau \wedge T} \beta(t)(c(t)-y(t)) \mathrm{d} t+\beta(\tau \wedge T)(W(\tau \wedge T)-n X(\tau \wedge T))\right] \leq W_{0}-n p_{0}, \\
\mathbb{E}_{\tau \wedge T}^{\mathbb{Q}_{\nu}}\left[\int_{\tau \wedge T}^{T} \beta(t) c(t) \mathrm{d} t+\beta(T) W(T)\right] \leq \beta(\tau \wedge T) W(\tau \wedge T)
\end{array}\right.
$$

for each $\psi \in \mathcal{P}$.

For each utility function $U_{i}(x), i=1,2$ and each $(s, t)$ such that $s \in[0, T]$ and $t \in[s, T]$, we denote by $I_{s}^{(i)}(x, t)$ the inverse function of

$$
\frac{\mathrm{d}}{\mathrm{d} x}\left[U_{i}(x) \exp \left\{-\int_{s}^{t} \rho(s) \mathrm{d} s\right\}\right]
$$

with respect to $x$. Similarly, for the function $V(x)$ defined in (7), we denote by $J(x)$ the inverse function of

$$
\frac{\mathrm{d}}{\mathrm{d} x}\left[V(x) \exp \left\{-\int_{0}^{\tau \wedge T} \rho(s) \mathrm{d} s\right\}\right]
$$

with respect to $x$. Under Assumption 2, for each $s, t$, the functions $I_{s}^{(i)}(x, t) \quad(i=1,2)$ and $J(x)$ exist, are continuous and strictly decreasing, and map $(0, \infty)$ onto itself. For each $s, t$, and $i$, we define the Legendre transformation $\tilde{u}_{s}(z, t)$ and $\tilde{V}$ by

$$
\begin{gathered}
\tilde{u}_{s}^{(i)}(z, t)=\sup _{c \geq 0}\left[\exp \left\{-\int_{s}^{t} \rho(u) \mathrm{d} u\right\} U_{i}(c)-z c\right], t \in[0, T], i=1,2, \\
\tilde{V}(z)=\sup _{w \geq 0}\left[\exp \left\{-\int_{0}^{\tau \wedge T} \rho(s) \mathrm{d} s\right\} V(w)-z w\right] .
\end{gathered}
$$

Then we can readily show that $I_{s}^{(i)}(0+, t)=\infty, I_{s}^{(i)}(\infty, t)=0, J(0+, t)=\infty, J(\infty, t)=0$, and

$$
\begin{gathered}
\tilde{u}_{s}^{(i)}(z, t)=\exp \left\{-\int_{s}^{t} \rho(u) \mathrm{d} u\right\} U_{i}\left(I_{s}^{(i)}(z, t)\right)-z I_{s}^{(i)}(z, t), t \in[0, T], i=1,2, \\
\tilde{V}(z)=\exp \left\{-\int_{0}^{\tau \wedge T} \rho(s) \mathrm{d} s\right\} V(J(z))-z J(z) .
\end{gathered}
$$

Now, in order to solve the problem (MP), we consider the following dual optimization problem: 


$$
\text { (DP) } \max _{n \in \mathbb{R}_{+}} \min _{\left(\zeta^{(n)}, \psi^{(n)}\right) \in \mathbb{R}_{++} \times \mathcal{P}} V_{0}\left(\zeta^{(n)}, \psi^{(n)}\right) \text {, }
$$

where

$$
V_{0}(\zeta, \psi)=\mathbb{E}\left[\int_{0}^{\tau \wedge T} \tilde{u}_{0}^{(1)}\left(\zeta \chi_{\psi}(t), t\right) \mathrm{d} t+\tilde{V}\left(\zeta \chi_{\psi}(\tau \wedge T)\right)+\zeta\left(W(0)-n p_{0}+\int_{0}^{\tau \wedge T} \chi_{\psi}(t) y(t) \mathrm{d} t+\chi_{\psi}(\tau \wedge T) n X(\tau \wedge T)\right)\right] .
$$

The household's optimal consumption/wealth process is given next:

Proposition 1 For a given $w>0$, Let $\mathcal{Z}(w)$ be a solution of the equation;

$$
w=\mathbb{E}_{\tau \wedge T}\left[\int_{\tau \wedge T}^{T} \chi(\tau \wedge T, t) I_{\tau \wedge T}^{(1)}(\mathcal{Z}(w) \chi(\tau \wedge T, t)) \mathrm{d} t+\chi(\tau \wedge T, T) I_{\tau \wedge T}^{(2)}(\mathcal{Z}(w) \chi(\tau \wedge T, T))\right]
$$

where, by recalling Equation (8),

$$
\chi(\tau \wedge T, t)=\frac{\beta(t) \chi^{B}(t)}{\beta(\tau \wedge T) \chi^{B}(\tau \wedge T)}, \quad t \in[\tau \wedge T, T] .
$$

Suppose that Assumptions 1 and 2 hold. Let $n^{*}$ be a solution to (DP) satisfying

$$
\begin{aligned}
& \mathbb{E}_{\tau \wedge T}\left[\int_{\tau \wedge T}^{T} \chi(\tau \wedge T, t) \times I_{\tau \wedge T}^{(1)}\left(\mathcal{Z}\left(J\left(\zeta^{*} \chi_{\psi^{*}}(\tau \wedge T)\right)\right) \chi(\tau \wedge T, t), t\right) \mathrm{d} t\right. \\
& \left.+\chi(\tau \wedge T, T) \times I_{\tau \wedge T}^{(2)}\left(\mathcal{Z}\left(J\left(\zeta^{*} \chi_{\psi^{*}}(\tau \wedge T)\right)\right) \chi(\tau \wedge T, T), T\right)\right]<\infty
\end{aligned}
$$

and

$$
\mathbb{E}^{\mathbb{Q}_{\psi^{*}}}\left[\int_{0}^{\tau \wedge T} \beta(t) I_{0}^{(1)}\left(\zeta^{*} \chi_{\psi^{*}}(t), t\right) \mathrm{d} t+\beta(\tau \wedge T) J\left(\zeta^{*} \chi_{\psi^{*}}(\tau \wedge T)\right)\right]<\infty,
$$

where $\left(\zeta^{*}, \psi^{*}\right):=\arg \min V_{0}\left(\zeta^{\left(n^{*}\right)}, \psi^{\left(n^{*}\right)}\right)$. Then, $n^{*}$ agrees with an optimal share $\hat{n}$ of the insurance policy in (MP) and an optimal consumption process $\hat{c}$ and the corresponding wealth process $\hat{W}$ are given, respectively, by

$$
\hat{c}(t)= \begin{cases}I_{0}^{(1)}\left(\zeta^{*} \chi_{\psi^{*}}(t), t\right) ; & t \in[0, \tau \wedge T) \\ I_{\tau \wedge T}^{(1)}\left(\mathcal{Z}\left(J\left(\zeta^{*} \chi_{\psi^{*}}(t)\right)\right) \chi(\tau \wedge T, t), t\right) ; & t \in[\tau \wedge T, T]\end{cases}
$$

and

$$
\hat{W}(t)= \begin{cases}\frac{1}{\beta(t)} \mathbb{E}_{t}^{\mathbb{Q}_{\psi^{*}}}\left[\int_{t}^{\tau \wedge T} \beta(s)(\hat{c}(s)-y(s)) \mathrm{d} s+\beta(\tau \wedge T)\left(\hat{W}(\tau \wedge T)-n^{*} X(\tau \wedge T)\right)\right] ; & t \in[0, \tau \wedge T), \\ \frac{1}{\beta(t)} \mathbb{E}_{t}^{\mathbb{Q}_{\psi^{*}}}\left[\int_{t}^{T} \beta(s) \hat{c}(s) d s+\beta(T) \hat{W}(T)\right] ; & t \in[\tau \wedge T, T]\end{cases}
$$

with $\hat{W}(\tau \wedge T)=J\left(\zeta^{*} \chi_{\psi^{*}}(\tau \wedge T)\right)$. Furthermore, $\zeta^{*}$ satisfies

$$
\begin{aligned}
& \mathbb{E}^{\mathbb{Q}_{\psi^{*}}}\left[\int_{0}^{\tau \wedge T} \beta(t) I_{0}^{(1)}\left(\zeta^{*} \chi_{\psi^{*}}(t), t\right) \mathrm{d} t+\beta(\tau \wedge T) \hat{W}(\tau \wedge T)\right] \\
& =W_{0}-n^{*} p_{0}+\mathbb{E}^{\mathbb{Q}_{\psi^{*}}}\left[\int_{0}^{\tau \wedge T} \beta(t) y(t) \mathrm{d} t+n^{*} \beta(\tau \wedge T) X(\tau \wedge T)\right] .
\end{aligned}
$$

In summary, we have obtained the following: If we assume that $n^{*}$ solves the dual problem (DP) along with $\left(\zeta^{*}, \psi^{*}\right)$, then $\zeta^{*}$ is represented by Equation (24). Moreover, $n^{*}$ is also the solution to the primal problem (MP), together with corresponding pair $(\hat{c}, \hat{W})$ that is given by Equations (22) and (23), respectively. However, in the problem (DP), it is quite difficult to derive optimal $\psi^{*}$ analytically. Hence, it is reasonable to consider conditions to guarantee $\psi^{*}=\lambda$. The next proposition provides one of such conditions.

Proposition 2 In addition to Assumptions 1 and 2, suppose that $I_{0}^{(1)}(x, t), t \in[0, T]$, and $J(x)$ are convex with respect to $x \in \mathbb{R}^{+}$, and that, for a sufficiently small $\varepsilon>0$, 


$$
\left|\mathbb{E}\left[I_{0}^{\prime(1)}\left(\chi_{\lambda}(t), t\right) \chi_{\lambda}(t)^{2}\right] \mathbb{E}\left[\left(\chi_{\psi}^{N}(t)^{2}-\chi_{\psi}^{N}(t)\right)\left(1_{\{\tau>t\}}+1_{\{\tau \in(t-, t]\}}\right)\right]\right|<\varepsilon,
$$

where $I_{0}^{\prime(1)}(x, t):=\frac{\mathrm{d}}{\mathrm{d} x} I_{0}^{(1)}(x, t)$. Then, the optimal solution $\left(\zeta^{*}, \psi^{*}\right)$ of the problem $(\mathbf{D P})$ is given by $\left(\zeta^{*}, \lambda\right)$. Especially, $\psi^{*}$ is uniquely given by $\lambda$.

We note the additional convexity assumption on $I_{0}^{(1)}(x, t), t \in[0, T]$, and $J(x)$ is satisfied by utility functions including logarithmic, etc., although implication of Equation (25) is not so clear.

Now in the next proposition, we will show how the optimal $\hat{n}\left(=n^{*}\right)$ looks like.

Proposition 3 Suppose that the assumptions of Proposition 2 hold. The optimal share $\hat{n}$ is given as follows. If $\mathbb{E}\left[\chi_{\lambda}(\tau \wedge T) X(\tau \wedge T)\right]>p_{0}$ then $\hat{n}=W_{0} / p_{0}$. Otherwise, if $\mathbb{E}\left[\chi_{\lambda}(\tau \wedge T) X(\tau \wedge T)\right]<p_{0}$, then $\hat{n}=0$, otherwise $\hat{n}$ is indefinite.

This proposition states that if the money received from the insurance contract at time $\tau$ is linear in $n$, the household either spends all of the initial endowment in the insurance contract or buys no insurance, depending on $\mathbb{E}\left[\chi_{\lambda}(\tau \wedge T) X(\tau \wedge T)\right] \gtrless p_{0}$. That is, depending on whether the discounted expected benefit from the insurance contract is greater or less than the insurance premium. This makes sense since the household tries to take a full advantage of possible mispricing in the insurance contract. On the other hand, if the insurance premium is priced in such a way $\mathbb{E}\left[\chi_{\lambda}(\tau \wedge T) X(\tau \wedge T)\right]=p_{0}$ (for example, based on the law of large numbers (LLN)), the household does not have a clue as to how it should determine an optimal $\hat{n}$.

The following result shows that we can explicitly derive the optimal portfolio process with an additional condition.

Proposition 4 Suppose that the assumptions of Proposition 2 hold. Provided that all parameters $r, \mu, \sigma$ and $\lambda$ are deterministic function of time $t \in[0, T]$, then the optimal portfolio process $\hat{\pi}=\{\hat{\pi}(t) ; t \in[0, T]\}$ is explicitly given by the following equations.

$$
\hat{\pi}(t)=-\frac{\zeta^{*} \chi(t) \xi(t)}{\sigma(t)} \times \mathbb{E}_{t}\left[\int_{t}^{\tau \wedge T} \chi(t, u)^{2} I_{0}^{\prime(1)}\left(\zeta^{*} \chi(u)\right) \mathrm{d} u+\chi(t, \tau \wedge T)^{2} J^{\prime}\left(\zeta^{*} \chi(\tau \wedge T)\right)\right] .
$$

Note that dependence on $n$ is implicit through Equation (24). The household need to decide the number of insurance contract first. Once given that $n$, they hold on to the optimal consumption Equation (22) and portfolio process Equation (26) to maximize their utility.

Our framework can be discussed in other settings. First, the insurance benefit was computed as a linear function of $n$ but we could assume a more general (non-linear) formula than in Equation (1). In this case, the optimal number of insurance contract can be an interior point between $\left(0, W_{0} / p_{0}\right)$. Secondly, our insurance premium $p_{0}$ here is given exogenously and Proposition 3 discusses the relationship between $p_{0}$ and the expected payment at $\tau$ whose form is basically LLN-based pricing. In this context, there exists an exceedingly increasing literature about pricing under distorted probabilities or Choquet pricing (see, for example, [24,25]). In conjunction with these new pricing schemes our problem can be extended to an equilibrium analysis between the household and the insurer.

\section{Acknowledgements}

We thank Masahiko Egami for his helpful comments and discussions. Hideki Iwaki is supported in part by Grant-in-Aid for Scientific Research (C) No. 22530310, Japan Society for the Promotion of Science.

\section{REFERENCES}

[1] M. O. Albizzati and H. Geman, "Interest Rate Risk Management and Valuation of the Surrender Option in Life Insurance Policies," Journal of Risk and Insurance, Vol. 61, No. 4, 1994, pp. 616-637. doi:10.2307/253641

[2] S. A. Persson and K. K. Aase, "Valuation of the Minimum Guaranteed Return Embedded in a Life Insurance Products," Journal of Risk and Insurance, Vol. 64, No. 4, 1997, pp. 599-617. doi:10.2307/253888

[3] M. J. Brennan and E. S. Schwartz, "The Pricing of Equity-linked Life Insurance Policies with an Asset Value Guarantee," Journal of Financial Economics, Vol. 3, No. 3, 1976, pp. 195-213. doi:10.1016/0304-405X(76)90003-9

[4] J. A. Nielsen and K. Sandman, "Equity-Linked Life Insurance: A Model with Stochastic Interest Rates," Insurance: Mathematics and Economics, Vol. 16, No. 3, 1995, pp. 225-253. doi:10.1016/0167-6687(95)00007-F

[5] E. Marceau and P. Gaillardetz, "On Life Insurance Reserves in a Stochastic Mortality and Interest Rates Environment," Insurance: Mathematics and Economics, Vol. 25, 1999, pp. 261-280. doi:10.1016/S0167-6687(99)00019-0

[6] A. R. Bacinello, "Equity Linked Life Insurance," In: E. Melnick and B. Everitt, Eds., Encyclopedia of Quantitative Risk Analysis and Assessment, John Wiley \& Sons, Hoboken, 2008. doi:10.1002/9780470061596.risk0346

[7] H. Iwaki, M. Kijima and Y. Morimoto, "An Economic Premium Principle in a Multiperiod Economy," Insurance: 
Mathematics and Insurance, Vol. 28, No. 3, 2001, pp. 325-339. doi:10.1016/S0167-6687(00)00081-0

[8] H. Iwaki, "An Economic Premium Principle in a Continuous-Time Economy," Journal of the Operations Research Society of Japan, Vol. 45, 2002, pp. 346-361.

[9] R. C. Merton, "Life Time Portfolio Selection under Uncertainty," Review of Economics and Statistics, Vol. 51, No. 3, 1969, pp. 247-257. doi:10.2307/1926560

[10] R. C. Merton, "Optimum Consumption and Portfolio Rules in a Continuous-time Model," Journal of Economic Theory, Vol. 3, No. 4, 1971, pp. 373-413. doi:10.1016/0022-0531(71)90038-X

[11] S. F. Richard, "Optimal Consumption, Portfolio and Life Insurance Rules for an Uncertain Lived Individual in a Continuous Time Model," Journal of Financial Economics, Vol. 2, No. 2, 1975, pp. 187-203. doi:10.1016/0304-405X(75)90004-5

[12] R. A. Campbell, "The Demand for Life Insurance: An Application of the Economics of Uncertainty," Journal of Finance, Vol. 35, No. 5, 1980, pp. 1155-1172. doi:10.1111/j.1540-6261.1980.tb02201.x

[13] D. F. Babbel and E. Ohtsuka, "Aspects of Optimal Multi-period Life Insurance," Journal of Risk and Insurance, Vol. 56, No. 3, 1989, pp. 460-481. doi: $10.2307 / 253168$

[14] Y. Zhu, "One-period Model of Individual Consumption, Life Insurance, and Investment Decisions," Journal of Risk and Insurance, Vol. 74, No. 3, 2007, pp. 613-636. doi:10.1111/j.1539-6975.2007.00227.x

[15] Z. Bodie, R. C. Merton and W. Samuelson, "Labor Supply Flexibility and Portfolio Choice in a Life Cycle Model," Journal of Economic Dynamics and Control, Vol. 16, No. 3-4, 1992, pp. 427-449. doi:10.1016/0165-1889(92)90044-F

[16] H. He and H. F. Pagès, "Labor Income, Borrowing Constraints and Equilibrium Asset Prices; A Duality Approach," Economic Theory, Vol. 3, No. 4, 1993, pp. 663696. doi:10.1007/BF01210265

[17] L. E. O. Svensson and I. M. Werner, "Nontradable Assets in Incomplete Markets: Pricing and Portfolio Choice," European Economic Review, Vol. 37, No. 5, 1993, pp.
1149-1168. doi:10.1016/0014-2921(93)90113-O

[18] I. Karatzas and S. E. Shreve, "Methods of Mathematical Finance," Springer-Verlag, New York, 1998.

[19] J. Cvitanić, W. Schachermayer and H. Wang, "Utility Maximization in Incomplete Markets with Random Endowment," Finance and Stochastics, Vol. 5, No. 2, 2001, pp. 259-272. doi:10.1007/PL00013534

[20] J. Grandell, "Double Stochastic Poisson Processes," Springer-Verlag, New York, 1976.

[21] A. Yashin and E. Arjas, "A Note on Random Intensities and Conditional Survival Functions," Journal of Applied Probability, Vol. 25, No. 3, 1988, pp. 630-635. doi: $10.2307 / 3213991$

[22] D. Kramkov and W. Schachermayer, "The Asymptotic Elasticity of Utility Functions and Optimal Investment in Incomplete Markets," Annals of Applied Probability, Vol. 9, No. 3, 1999, pp. 904-950. doi:10.1214/aoap/1029962818

[23] N. Bellamy and M. Jeanblanc, "Incompleteness of Markets Driven by a Mixed Diffusion," Finance and Stochastics, Vol. 4, No. 2, 2000, pp. 209-222. doi: $10.1007 / \mathrm{s} 007800050012$

[24] S. Wang, V. R. Young and H. Panjier, "Axiomatic Characterization of Insurance Prices," Insurence: Mathematics and Economics, Vol. 21, No. 2, 1997, pp.173-183. doi:10.1016/S0167-6687(97)00031-0

[25] V. R. Young and T. Zariphopoulou, "Computation of Distorted Probabilities for Diffusion Processes via Stochastic Control Methods," Insurance: Mathematics and Economics, Vol. 27, No. 1, 2000, pp. 1-18. doi:10.1016/S0167-6687(99)00061-X

[26] D. Cuoco, "Optimal Consumption and Equilibrium Prices wiht Portfolio Constraints and Stochastic Income," Journal of Economic Theory, Vol. 72, No. 1, 1997, pp. 33-73. doi:10.1006/jeth.1996.2207

[27] J. Cvitanić and I. Karatzas, "Convex Duality in Constrained Portfolio Optimization," Annals of Applied Probability, Vol. 2, No. 4, 1992, pp. 767-818. doi:10.1214/aoap/1177005576

[28] R. T. Rockafellar, "Convex Analysis," Princeton University Press, New Jersey, 1970. 


\section{Appendix}

\section{Proof of Lemma 1:}

For any $\psi \in \mathcal{P}$, suppose that $(c, W)$ is in $\mathcal{C}$. Then, from Equation (3) and Itô's formula, we obtain

$$
\beta(t) W(t)= \begin{cases}W_{0}-n p_{0}+\int_{0}^{t} \beta(s)(y(s)-c(s)) \mathrm{d} s+\int_{0}^{t} \beta(s) \pi(s) \sigma(s) \mathrm{d} \tilde{B} & \text { if } t \in[0, \tau \wedge T), \\ \beta(\tau)(W(\tau-)+n X(\tau))-\int_{\tau}^{t} \beta(s) c(s) \mathrm{d} s+\int_{\tau}^{t} \beta(s) \pi(s) \sigma(s) \mathrm{d} \tilde{B} & \text { if } t \in[\tau \wedge T, T],\end{cases}
$$

where $\tilde{B}(t):=B(t)+\int_{0}^{t} \xi(s) \mathrm{d} s$ is a standard Brownian motion under $\mathbb{Q}_{\psi}$ for all $\psi \in \mathcal{P}$. Now, on the set $\{\tau<T\}$, in the first equation, set $t=\tau-$ and note that $W(\tau)=W(\tau-)+n X(\tau)$ and in the second equation, set $t=T$. Then we obtain Equation (15) after taking the expectation for this set $\{\tau<T\}$. Similarly, on the set $\{\tau \geq T\}$, in the first equation, we set $t=T-$ and note that $W(T)=W(T-)+n X(T) \quad($ recall Assumption 1). In the second equation, we set $t=\tau=T$ (recall Assumption 1 again). On this set we also obtain Equation (15) after taking the conditional expectation.

Proof of Proposition 1:

The proof here adapts the arguments in [26,27]. Assume that $\left(\zeta^{*}, \psi^{*}\right)$ solves (DP) along with $n^{*}$, and that

$$
\begin{aligned}
& J\left(\zeta^{*} \chi_{\psi^{*}}(\tau \wedge T)\right) \\
& =\mathbb{E}_{\tau \wedge T}\left[\int_{\tau \wedge T}^{T} \chi(\tau \wedge T, t) \times I_{\tau \wedge T}^{(1)}\left(\mathcal{Z}\left(J\left(\zeta^{*} \chi_{\psi^{*}}(\tau \wedge T)\right)\right) \chi(\tau \wedge T, t), t\right) \mathrm{d} t\right. \\
& \left.+\chi(\tau \wedge T, T) \times I_{\tau \wedge T}^{(2)}\left(\mathcal{Z}\left(J\left(\zeta^{*} \chi_{\psi^{*}}(\tau \wedge T)\right)\right) \chi(\tau \wedge T, T), T\right)\right]<\infty, \\
& \mathbb{E}\left[\int_{0}^{\tau \wedge T} \chi_{\psi^{*}}(t) I_{0}^{(1)}\left(\zeta^{*} \chi_{\psi^{*}}(t), t\right) \mathrm{d} t+\chi_{\psi^{*}}(\tau \wedge T) J\left(\zeta^{*} \chi_{\psi^{*}}(\tau \wedge T)\right)\right]<\infty
\end{aligned}
$$

holds. In order to prove that $(\hat{c}, \hat{W}(T))$ in Equations (22) and (23) is optimal, we will proceed in two steps; first we will show that

$$
\begin{aligned}
& \mathbb{E}\left[\int_{0}^{\tau \wedge T} \exp \left\{-\int_{0}^{t} \rho(s) \mathrm{d} s\right\} U_{1}(\hat{c}(t)) \mathrm{d} t+\exp \left\{-\int_{0}^{\tau \wedge T} \rho(t) \mathrm{d} t\right\} V(\hat{W}(\tau \wedge T))\right] \\
& \geq \mathbb{E}\left[\int_{0}^{\tau \wedge T} \exp \left\{-\int_{0}^{t} \rho(s) \mathrm{d} s\right\} U_{1}(c(t)) \mathrm{d} t+\exp \left\{-\int_{0}^{\tau \wedge T} \rho(t) \mathrm{d} t\right\} V(W(\tau \wedge T))\right]
\end{aligned}
$$

and

$$
\begin{aligned}
V(\hat{W}(\tau \wedge T)) & =\mathbb{E}_{\tau \wedge T}\left[\int_{\tau \wedge T}^{T} \exp \left\{-\int_{\tau \wedge T}^{t} \rho(s) \mathrm{d} s\right\} U_{1}(\hat{c}(t)) \mathrm{d} t+\exp \left\{-\int_{\tau \wedge T}^{T} \rho(t) \mathrm{d} t\right\} U_{2}(\hat{W}(T))\right] \\
& \geq \mathbb{E}_{\tau \wedge T}\left[\int_{\tau \wedge T}^{T} \exp \left\{-\int_{\tau \wedge T}^{t} \rho(s) \mathrm{d} s\right\} U_{1}(c(t)) \mathrm{d} t+\exp \left\{-\int_{\tau \wedge T}^{T} \rho(t) \mathrm{d} t\right\} U_{2}(W(T))\right]
\end{aligned}
$$

hold for all $(c, W(T)) \in \mathcal{C}$, and then that $(\hat{c}, \hat{W}(T)) \in \mathcal{C}$.

Step 1 (optimality). By Assumption 2-(2), there exists $a, b \in(0, \infty)$ such that for each $i=1,2$,

$$
a U_{i}^{\prime}\left(I_{s}^{(i)}(z, t)\right) \geq U_{i}^{\prime}\left(b I_{s}^{(i)}(z, t)\right), t \in[s, T], s \in[0, T] .
$$

Applying $I_{s}^{(i)}(\cdot, t)$ to both sides and iterating, show that for all $a \in(0, \infty)$ there exists a $b \in(0, \infty)$ such that

$$
\begin{aligned}
& I_{s}^{(i)}(a z, t) \leq b I_{s}^{(i)}(z, t), \\
& (z, t) \in(0, \infty) \times[s, T], s \in[0, T] .
\end{aligned}
$$

Hence Equation (28) implies

$$
\mathbb{E}\left[\int_{0}^{\tau \wedge T} \chi_{\psi^{*}}(t) I_{0}^{(1)}\left(\zeta_{1} \chi_{\psi^{*}}(t), t\right) \mathrm{d} t+\chi_{\psi^{*}}(\tau \wedge T) J\left(\zeta_{2} \chi_{\psi^{*}}(\tau \wedge T)\right)\right]<\infty
$$

for all $\zeta_{i} \in(0, \infty), i=1,2$.

By the optimality of $\zeta^{*}$, we have 


$$
\begin{aligned}
0= & \lim _{\epsilon \rightarrow 0} \frac{V_{0}\left(\zeta^{*}+\epsilon, \psi^{*}\right)-V_{0}\left(\zeta^{*}, \psi^{*}\right)}{\epsilon}=\mathbb{E}\left[\int_{0}^{\tau \wedge T} \lim _{\epsilon \rightarrow 0}\left(\tilde{u}_{1}\left(\left(\zeta^{*}+\epsilon\right) \chi_{\psi^{*}}(t), t\right)-\tilde{u}_{1}\left(\zeta^{*} \chi_{\psi^{*}}(t), t\right)\right) / \epsilon \mathrm{d} t\right. \\
& +\lim _{\epsilon \rightarrow 0}\left(\tilde{V}\left(\left(\zeta^{*}+\epsilon\right) \chi_{\psi^{*}}(\tau \wedge T), \tau \wedge T\right)-\tilde{V}\left(\zeta^{*} \chi_{\psi^{*}}(\tau \wedge T), \tau \wedge T\right)\right) / \epsilon+W_{0}-n p_{0} \\
& \left.+\int_{0}^{\tau \wedge T} \chi_{\psi^{*}}(t) y(t) \mathrm{d} t+n \chi(\tau \wedge T) X(\tau \wedge T)\right] \\
= & W_{0}-n p_{0}-\mathbb{E}\left[\int_{0}^{\tau \wedge T} \chi_{\psi^{*}}(t)(\hat{c}(t)-y(t)) \mathrm{d} t+\chi_{\psi^{*}}(\tau \wedge T)(\hat{W}(\tau \wedge T)-n X(\tau \wedge T))\right],
\end{aligned}
$$

where the second equality follows the dominated convergence theorem, using Equation (31) and the fact that

$$
\begin{aligned}
& \left|\frac{\tilde{u}_{0}^{(1)}\left(\left(\zeta^{*}+\epsilon\right) \chi_{\psi^{*}}(t), t\right)-\tilde{u}_{0}^{(1)}\left(\zeta^{*} \chi_{\psi^{*}}(t), t\right)}{\epsilon}\right| \leq \frac{\tilde{u}_{0}^{(1)}\left(\left(\zeta^{*}-|\epsilon|\right) \chi_{\psi^{*}}(t), t\right)-\tilde{u}_{0}^{(1)}\left(\zeta^{*} \chi_{\psi^{*}}(t), t\right)}{|\epsilon|} \\
& \leq \chi_{\psi^{*}}(t) I_{0}^{(1)}\left(\left(\zeta^{*}-|\epsilon|\right) \chi_{\psi^{*}}(t), t\right) \leq \chi_{\psi^{*}}(t) I_{0}^{(1)}\left(\frac{\zeta^{*}}{2} \chi_{\psi^{*}}(t), t\right),
\end{aligned}
$$

and that

$$
\begin{aligned}
& \left|\left(\tilde{V}\left(\left(\zeta^{*}+\epsilon\right) \chi_{\psi^{*}}(t), \tau \wedge T\right)-\tilde{V}\left(\zeta^{*} \chi_{\psi^{*}}(\tau \wedge T), \tau \wedge T\right)\right) / \epsilon\right| \\
& \leq\left(\tilde{V}\left(\left(\zeta^{*}-|\epsilon|\right) \chi_{\psi^{*}}(\tau \wedge T), \tau \wedge T\right)-\tilde{u}_{i}\left(\zeta^{*} \chi_{\psi^{*}}(\tau \wedge T), \tau \wedge T\right)\right) / \epsilon \mid \\
& \leq \chi_{\psi^{*}}(t) J\left(\left(\zeta^{*}-|\epsilon|\right) \chi_{\psi^{*}}(\tau \wedge T), \tau \wedge T\right) \leq \chi_{\psi^{*}}(t) J\left(\frac{\zeta^{*}}{2} \chi_{\psi^{*}}(\tau \wedge T), \tau \wedge T\right) \forall|\epsilon|<\frac{\zeta^{*}}{2},
\end{aligned}
$$

because both $\tilde{u}_{0}^{(i)}(\cdot, t)$ and $\tilde{V}(\cdot, \tau \wedge T)$ are decreasing and convex,

$$
\frac{\partial}{\partial z} \tilde{u}_{0}^{(1)}(z, t)=-I_{0}^{(i)}(z, t), \frac{\partial}{\partial z} \tilde{V}(z, \tau \wedge T)=-J(z, \tau \wedge T),
$$

and both $I_{0}^{(1)}(\cdot, t)$ and $J(\cdot, \tau \wedge T)$ are decreasing). Since by concavity

$$
\begin{gathered}
\exp \left\{-\int_{0}^{t} \rho(s) \mathrm{d} s\right\} U_{1}\left(I_{0}^{(1)}(z, t)\right)-\exp \left\{-\int_{0}^{t} \rho(s) \mathrm{d} s\right\} U_{1}(c) \geq z\left[I_{0}^{(1)}(z, t)-c\right], \\
\exp \left\{-\int_{0}^{\tau \wedge T} \rho(s) \mathrm{d} s\right\} V(J(z))-\exp \left\{-\int_{0}^{\tau \wedge T} \rho(s) \mathrm{d} s\right\} V(c) \geq z[J(z)-c] \forall c>0, z>0,
\end{gathered}
$$

it then, by evaluating the previous inequality at $z=\zeta^{*} \chi_{\psi^{*}}(t)$ and using the definition of $\hat{c}$ and $\hat{W}(\tau \wedge T)$ in Equations (22) and (23), follows from Equations (15) and (32)

$$
\begin{aligned}
& \mathbb{E}\left[\int_{0}^{\tau \wedge T} \exp \left\{-\int_{0}^{t} \rho(s) \mathrm{d} s\right\} U_{1}(\hat{c}(t)) \mathrm{d} t+\exp \left\{-\int_{0}^{\tau \wedge T} \rho(t) \mathrm{d} t\right\} V(\hat{W}(\tau \wedge T))\right] \\
& -\mathbb{E}\left[\int_{0}^{\tau \wedge T} \exp \left\{-\int_{0}^{t} \rho(s) \mathrm{d} s\right\} U_{1}(c(t)) \mathrm{d} t+\exp \left\{-\int_{0}^{\tau \wedge T} \rho(t) \mathrm{d} t\right\} V(W(\tau \wedge T))\right] \\
& \geq \zeta^{*} \mathbb{E}\left[\int_{0}^{\tau \wedge T} \chi_{\psi^{*}}(t)(\hat{c}(t)-c(t)) \mathrm{d} t+\chi_{\psi^{*}}(\tau \wedge T)(\hat{W}(\tau \wedge T)-W(\tau \wedge T))\right] \geq 0 .
\end{aligned}
$$

Furthermore, we can easily confirm that Equation (30) holds since Equation (18), Equation (21) and that $\hat{W}(\tau \wedge T)=J\left(\zeta^{*} \chi_{\psi^{*}}(\tau \wedge T)\right)$. Hence, $(\hat{c}, \hat{W}(\tau \wedge T))$ must be optimal provided it is in $\mathcal{C}$.

Step 2 (feasibility). We are only left to show that there exists an admissible portfolio process $\hat{\pi}$ financing $(\hat{c}, \hat{W}(T))$. For any $n \in \mathbb{R}_{+}$, define a process $\tilde{W}=\{\tilde{W}(t) ; t \in[0, \tau \wedge T]\}$ by

$$
\tilde{W}(t):= \begin{cases}\tilde{W}(t-)+n X(t) & \text { if } t=\tau \wedge T, \\ \tilde{W}(t-) & \text { if } t \in(0, \tau \wedge T),\end{cases}
$$

where 


$$
\begin{aligned}
\tilde{W}(t-) & :=\chi_{\psi^{*}}(t-)^{-1} \mathbb{E}_{t-}\left[\int_{t}^{\tau \wedge T} \chi_{\psi^{*}}(s)(\hat{c}(s)-y(s)) \mathrm{d} s+\chi_{\psi^{*}}(\tau \wedge T)(\hat{W}(\tau \wedge T)-n X(\tau \wedge T))\right] \\
& =\beta(t)^{-1} \mathbb{E}_{t-}^{Q_{\psi^{*}}}\left[\int_{t}^{\tau \wedge T} \beta(s)(\hat{c}(s)-y(s)) \mathrm{d} s+\beta(\tau \wedge T)(\hat{W}(\tau \wedge T)-n X(\tau \wedge T))\right],
\end{aligned}
$$

Clearly, $\tilde{W}(\tau \wedge T)=\hat{W}(\tau \wedge T)$, and $\tilde{W}$ is bounded below (because of boundedness of $y$ and $X$ by the ground assumption). Also, it follows from the martingale representation theorem that there exists a process $\left\{\left(\theta_{1}(t), \theta_{2}(t)\right) ; t \in[0, \tau \wedge T]\right\}$ with

such that,

$$
\int_{0}^{\tau \wedge T}\left|\theta_{1}(t)^{2}+\theta_{2}(t)\right| \mathrm{d} t<\infty
$$

$$
\begin{aligned}
& \beta(t) \tilde{W}(t-)+\int_{0}^{t} \beta(s)(\hat{c}(s)-y(s)) \mathrm{d} s \\
& =\tilde{W}(0)+\int_{0}^{t} \theta_{1}(s) \mathrm{d} \tilde{B}(s) \\
& +\int_{0}^{t} \theta_{2}(s)\left(\mathrm{d} N(s)-\psi^{*}(s) \mathrm{d} s\right), \quad t \in(0, \tau \wedge T] .
\end{aligned}
$$

$$
\begin{aligned}
\tilde{W}(t)= & \tilde{W}(0)+\int_{0}^{t}(r(s) \tilde{W}(s)+y(s)-\hat{c}(s)) \mathrm{d} s+\int_{0}^{t} \beta(s)^{-1} \theta_{1}(s) \mathrm{d} \tilde{B}(s)+n X(t) 1_{\{t=\tau \wedge T\}} \\
& +\int_{0}^{t} \beta(s)^{-1} \theta_{2}(s)\left(\mathrm{d} N(s)-\psi^{*}(s) \mathrm{d} s\right) \\
= & \tilde{W}(0)+\int_{0}^{t}(r(s) \tilde{W}(s)+y(s)-\hat{c}(s)) \mathrm{d} s+\int_{0}^{t} \pi(s)[(\mu(s)-r(s)) \mathrm{d} s+\sigma(s) \mathrm{d} B(s)] \\
& +n X(t) 1_{\{t=\tau \wedge T\}}+\int_{0}^{t} \beta(s)^{-1} \theta_{2}(s)\left(\mathrm{d} N(s)-\psi^{*}(s) \mathrm{d} s\right) .
\end{aligned}
$$

A comparison with Equation (3) then reveals that only if we verify that $\theta_{2}(t)=0$ for all $t \in[0, \tau \wedge T]$ with $\hat{W}(t)=\tilde{W}(t)$, the proof will be completed.

For this purpose, fix an arbitrary $\psi=\{\psi(t) ; t \in[0, \tau \wedge T]\} \in \mathcal{P}$ and define stochastic processes $\kappa$ and $\bar{\kappa}$. by, respectively,

$$
\kappa(t):=\int_{0}^{t} \frac{\psi^{*}(s)-\psi(s)}{\psi^{*}(s)}\left(\mathrm{d} N(s)-\psi^{*}(s) \mathrm{d} s\right)
$$

and

$$
\bar{\kappa}(t):=\int_{0}^{t}\left(\psi^{*}(s)-\psi(s)\right) \mathrm{d} s, \quad t \in[0, \tau \wedge T],
$$

as well as the sequence of stopping times

$$
\tau_{m}:=\inf \left\{t \in[0, \tau \wedge T]:|\kappa(t)|+|\bar{\kappa}(t)|+\left|\frac{\psi^{*}(t)-\psi(t)}{\psi^{*}(t)}\right| \geq m\right\}, m=1,2, \cdots
$$

Then $\tau_{m} \uparrow \tau \wedge T$. Also, letting

$$
\psi_{\epsilon, m}(t):=\psi^{*}(t)+\epsilon\left[\psi(t)-\psi^{*}(t)\right] 1_{\left\{t \leq \tau_{m}\right\}}
$$

for $\epsilon \in(0,1 /(m l))$ with some $l>1$. Clearly

$$
\begin{aligned}
& \int_{0}^{\tau \wedge T} \psi_{\epsilon, m}(t) \mathrm{d} t<\infty \text { a.s. } \\
& \epsilon \zeta^{*} \cdot Y_{m}^{\epsilon}:=\int_{0}^{\tau \wedge T}\left[\tilde{u}_{0}^{(1)}\left(\zeta^{*} \chi_{\psi_{\epsilon, m}}(t), t\right)-\tilde{u}_{0}^{(1)}\left(\zeta^{*} \chi_{\psi^{*}}(t), t\right)\right] \mathrm{d} t+\tilde{V}\left(\zeta^{*} \chi_{\psi_{\epsilon, m}}(\tau \wedge T)\right)-\tilde{V}\left(\zeta^{*} \chi_{\psi^{*}}(\tau \wedge T)\right) \\
& +\zeta^{*} \int_{0}^{\tau \wedge T}\left[\chi_{\psi_{\epsilon, m}}(t)-\chi_{\psi^{*}}(t)\right] y(t) \mathrm{d} t+\zeta^{*}\left[\chi_{\psi_{\epsilon, m}}(\tau \wedge T)-\chi_{\psi^{*}}(\tau \wedge T)\right] n X(\tau \wedge T) .
\end{aligned}
$$

Hence $\psi_{\epsilon, m}=\left\{\psi_{\epsilon, m}(t) ; t \in[0, \tau \wedge T]\right\}$ belongs to $\mathcal{P}$. Therefore, as in Equation (14), we can define $\chi_{\psi_{\epsilon, m}}$ and consider

$$
0 \leq \frac{1}{\epsilon \zeta^{*}}\left[V_{0}\left(\zeta^{*}, \psi_{\epsilon, m}\right)-V_{0}\left(\zeta^{*}, \psi^{*}\right)\right]=\mathbb{E}\left[Y_{m}^{\epsilon}\right]
$$

Introduce the ratio 
We have then

$$
\begin{aligned}
R^{\epsilon}(t) & :=\frac{\chi_{\psi_{\epsilon, m}}(t)}{\chi_{\psi^{*}}(t)}=\exp \left\{\int_{0}^{t} \ln \left(\frac{\psi_{\epsilon, m}(s)}{\psi^{*}(s)}\right) \mathrm{d} N(s)-\int_{0}^{t}\left(\psi_{\epsilon, m}(s)-\psi^{*}(s)\right) \mathrm{d} s\right\} \\
& =\exp \left\{\epsilon \bar{\kappa}\left(t \wedge \tau_{m}\right)\right\}\left(1-\epsilon\left(\kappa\left(t \wedge \tau_{m}\right)+\bar{\kappa}\left(t \wedge \tau_{m}\right)\right)\right), t \in[0, \tau \wedge T] .
\end{aligned}
$$

$$
0<\exp \left\{-\frac{1}{l}\right\}\left(1-\frac{1}{l}\right) \leq \exp \{-\epsilon m\}(1-\epsilon m) \leq R^{\epsilon}(t) \leq \exp \{\epsilon m\}(1+\epsilon m) \leq \exp \left\{\frac{1}{l}\right\}\left(1+\frac{1}{l}\right),
$$

as well as the upper bounds for the random variable $Y_{m}^{\epsilon}$ :

$$
Y_{m}^{\epsilon} \leq Q_{m}, \quad Y_{m}^{\epsilon} \leq Y_{m},
$$

where

$$
\begin{aligned}
Q_{m}:= & \int_{0}^{\tau \wedge T} \chi_{\psi^{*}}(t) \frac{1-R^{\epsilon}(t)}{\epsilon} \times I_{0}^{(1)}\left(\zeta^{*} \exp \{-\operatorname{sgn}(t) \epsilon m\}(1-\operatorname{sgn}(t) \epsilon m) \chi_{\psi^{*}}(t), t\right) \mathrm{d} t \\
& +\chi_{\psi^{*}}(\tau \wedge T) \frac{1-R^{\epsilon}(\tau \wedge T)}{\epsilon} \times J\left(\zeta^{*} \exp \{-\operatorname{sgn}(\tau \wedge T) \epsilon m\}(1-\operatorname{sgn}(\tau \wedge T) \epsilon m) \times \chi_{\psi^{*}}(\tau \wedge T)\right) \\
& -\int_{0}^{\tau \wedge T} \chi_{\psi^{*}}(t) \frac{1-R^{\epsilon}(t)}{\epsilon} y(t) \mathrm{d} t-\chi_{\psi^{*}}(\tau \wedge T) \frac{1-R^{\epsilon}(\tau \wedge T)}{\epsilon} n X(\tau \wedge T), \\
Y_{m}:= & \sup _{\epsilon \in(0,1 / m l)} \frac{1-\exp \{-\epsilon m\}(1-\epsilon m)}{\epsilon} \times\left[\int_{0}^{\tau \wedge T} \chi_{\psi^{*}}(t) \times I_{0}^{(1)}\left(\zeta^{*} \exp \left\{-\frac{1}{l}\right\}\left(1-\frac{1}{l}\right) \chi_{\psi^{*}}(t), t\right) \mathrm{d} t\right. \\
& \left.+\chi_{\psi^{*}}(T) J\left(\zeta^{*} \exp \left\{-\frac{1}{l}\right\}\left(1-\frac{1}{l}\right) \chi_{\psi^{*}}(\tau \wedge T)\right)+\int_{0}^{\tau \wedge T} \chi_{\psi^{*}}(t) y(t) \mathrm{d} t+\chi_{\psi^{*}}(\tau \wedge T) n X(\tau \wedge T)\right],
\end{aligned}
$$

and

$$
\operatorname{sgn}(t)= \begin{cases}1 & \text { if } 1-R^{\epsilon}(t) \geq 0 \\ -1 & \text { if } 1-R^{\epsilon}(t)<0 .\end{cases}
$$

$$
J(y)=-\frac{\partial}{\partial y} \tilde{V}(y)
$$

We have used the mean-value theorem applying to

$$
\begin{aligned}
& I_{0}^{(1)}(y, t)=-\frac{\partial}{\partial y} \tilde{u}_{0}^{(1)}(y, t) \\
& 0 \leq \varlimsup_{\epsilon \downarrow 0} \mathbb{E}\left[Y_{m}^{\epsilon}\right] \leq \mathbb{E}\left[\varlimsup_{\epsilon \downarrow 0} Y_{m}^{\epsilon}\right] \leq \mathbb{E}\left[\varlimsup_{\epsilon \downarrow 0} Q_{m}^{\epsilon}\right] \\
& =\mathbb{E}\left[\int_{0}^{\tau \wedge T} \chi_{\psi^{*}}(t) \kappa\left(t \wedge \tau_{m}\right)(\hat{c}(t)-y(t)) \mathrm{d} t+\chi_{\psi^{*}}(\tau \wedge T) \kappa\left(\tau \wedge \tau_{m}\right) \times(\hat{W}(\tau \wedge T)-n X(\tau \wedge T))\right] \\
& =\mathbb{E}\left[\int_{0}^{\tau \wedge \tau_{m}} \chi_{\psi^{*}}(t) \kappa(t)(\hat{c}(t)-y(t)) \mathrm{d} t\right]+\kappa\left(\tau \wedge \tau_{m}\right) \\
& \times \mathbb{E}_{\tau \wedge \tau_{m}}\left[\int_{\tau \wedge \tau_{m}}^{\tau \wedge T} \chi_{\psi^{*}}(t)(\hat{c}(t)-y(t)) \mathrm{d} t+\chi_{\psi^{*}}(\tau \wedge T)(\hat{W}(\tau \wedge T)-n X(\tau \wedge T))\right] \\
& =\mathbb{E}\left[\int_{0}^{\tau \wedge \tau_{m}} \chi_{\psi^{*}}(t) \kappa(t)(\hat{c}(t)-y(t)) \mathrm{d} t+\kappa\left(\tau \wedge \tau_{m}\right) \chi_{\psi^{*}}\left(\tau \wedge \tau_{m}\right) \hat{W}\left(\tau \wedge \tau_{m}\right)\right] .
\end{aligned}
$$

We used the definition of $\hat{W}(t)$ Equation (23) in the third equality. On the other hand, using Equations (33)-(36) with $\tilde{W}=\hat{W}$ and Itô's lemma shows that

$$
\begin{aligned}
& \beta\left(\tau \wedge \tau_{m}\right) \kappa\left(\tau \wedge \tau_{m}\right) \hat{W}\left(\tau \wedge \tau_{m}\right)+\int_{0}^{\tau \wedge \tau_{m}} \beta(s) \kappa(s)(\hat{c}(s)-y(s)) \mathrm{d} s \\
& =\int_{0}^{\tau \wedge \tau_{m}} \beta(s) \hat{W}(s) \frac{\psi^{*}(s)-\psi(s)}{\psi^{*}(s)} \times\left[\mathrm{d} N(s)-\psi^{*}(s) \mathrm{d} s\right]+\int_{0}^{\tau \wedge \tau_{m}} \beta(s) \kappa(s) \theta_{2}(s)\left(\mathrm{d} N(s)-\psi^{*}(s) \mathrm{d} s\right) \\
& +\int_{0}^{\tau \wedge \tau_{m}} \beta(s) \kappa(s) \pi(s) \sigma(s) \mathrm{d} \tilde{B}(s)+\int_{0}^{\tau \wedge \tau_{m}} \beta(s) \theta_{2}(s) \frac{\psi^{*}(s)-\psi(s)}{\psi^{*}(s)} \mathrm{d} N(s) .
\end{aligned}
$$


Therefore we have

$$
\begin{aligned}
& \mathbb{E}\left[\int_{0}^{\tau \wedge \tau_{m}} \chi_{\psi^{*}}(s) \kappa(s)(\hat{c}(s)-y(s)) \mathrm{d} s\right. \\
& \left.+\chi_{\psi^{*}}\left(\tau \wedge \tau_{m}\right) \kappa\left(\tau \wedge \tau_{m}\right) \hat{W}\left(\tau \wedge \tau_{m}\right)\right] \\
& =\mathbb{E}\left[\int_{0}^{\tau \wedge \tau_{m}} \chi_{\psi^{*}}(s) \theta_{2}(s)\left(\psi^{*}(s)-\psi(s)\right) \mathrm{d} s\right] .
\end{aligned}
$$

Substituting Equation (38) into Equation (37) gives

$$
\begin{aligned}
0 & \leq \lim _{\epsilon \downarrow 0} \frac{V_{0}\left(\zeta^{*}, \psi_{\epsilon, m}\right)-V_{0}\left(\zeta^{*}, \psi^{*}\right)}{\zeta^{*} \epsilon} \\
& \leq \mathbb{E}\left[\int_{0}^{\tau \wedge \tau_{m}} \chi_{\psi^{*}}(s) \theta_{2}(s)\left(\psi^{*}(s)-\psi(s)\right) \mathrm{d} s\right] .
\end{aligned}
$$

Taking $\psi$ as $\psi=\psi^{*}+\eta$ with $\eta \in \mathcal{P}$, it follows that

$$
\begin{aligned}
& \mathbb{E}\left[\int_{0}^{\tau \wedge \tau_{m}} \chi_{\psi^{*}}(s) \theta_{2}(s) \eta(s) \mathrm{d} s\right] \leq 0 . \\
M & :=\min _{\psi \in \mathcal{P}} V_{0}(\zeta, \psi) \\
= & \mathbb{E}\left[\int_{0}^{\tau \wedge T} \tilde{u}_{0}^{(1)}\left(\zeta \chi_{\psi}(t), t\right) \mathrm{d} t+\tilde{V}\left(\zeta \chi_{\psi}(\tau \wedge T)\right)\right]+\zeta\left(W_{0}-n p_{0}+\mathbb{E}\left[\int_{0}^{\tau \wedge T} y(t) \chi_{\psi}(t) \mathrm{d} t+n \chi_{\psi}(\tau \wedge T) X(\tau \wedge T)\right]\right) .
\end{aligned}
$$

Equation (39) leads to a stronger statement:

$$
\theta_{2}(t) \eta(t) \leq 0, \quad t \in[0, \tau \wedge T] .
$$

Indeed, suppose that, for some $t \in[0, \tau \wedge T]$, the set $E=\left\{\omega \in \Omega ; \theta_{2}(t) \eta(t)>0\right\}$ had positive probability for some $\eta \in \mathcal{P}$ such that $\psi^{*}+\eta \in \mathcal{P}$. Then by selecting $\eta^{\prime}=\eta 1_{E}$, we have $\eta^{\prime} \in \mathcal{P}, \psi^{*}+\eta^{\prime} \in \mathcal{P}$, and

$$
\mathbb{E}\left[\int_{0}^{\tau \wedge \tau_{m}} \chi_{\psi^{*}}(s) \theta_{2}(s) \eta^{\prime}(s) \mathrm{d} s\right]>0,
$$

contradicting Equation (39). From Theorem 13.1 of [28] and Equation (40), we can conclude that $\theta_{2}(t)=0$, $t \in[0, \tau \wedge T]$.

\section{Proof of Proposition 2:}

We first show that if a solution $\left(\zeta^{*}, \psi^{*}\right)$ exists, then this solution is unique with respect to $\psi^{*}$. Let $\hat{\psi}$ be

Since we can readily show that $\tilde{V}(y)$ and $\tilde{u}_{0}^{(1)}(y, t), t \in[0, T]$, are convex in $y$,

$$
\begin{aligned}
& M \geq \mathbb{E}\left[\int_{0}^{\tau \wedge T} \tilde{u}_{0}^{(1)}\left(\eta \zeta \chi_{\psi^{*}}(t)+(1-\eta) \zeta \chi_{\hat{\psi}}(t), t\right) \mathrm{d} t+\tilde{V}\left(\eta \zeta \chi_{\psi^{*}}(\tau \wedge T)+(1-\eta) \zeta \chi_{\hat{\psi}}(\tau \wedge T)\right)\right] \\
& +\zeta\left(W_{0}-n p_{0}+\mathbb{E}\left[\int_{0}^{\tau \wedge T} y(t)\left(\eta \chi_{\psi^{*}}(t)+(1-\eta) \chi_{\hat{\psi}}(t)\right) \mathrm{d} t+n\left(\eta \chi_{\psi^{*}}(\tau \wedge T)+(1-\eta) \chi_{\hat{\psi}}(\tau \wedge T)\right) X(\tau \wedge T)\right]\right), \eta \in[0,1] .
\end{aligned}
$$

Since $M$ is the minimum, we have equality in Equation (42). By the previous inequality, we conclude that $\psi^{*}=\hat{\psi}$. To identify the optimal $\psi$, we consider an equivalent problem. Let us define

$$
\begin{aligned}
& G(\eta):=\mathbb{E}\left[\int_{0}^{\tau \wedge T} \tilde{u}_{0}^{(1)}\left(\eta \zeta \chi_{\psi^{*}}(t)+(1-\eta) \zeta \chi_{\psi}(t), t\right) \mathrm{d} t+\tilde{V}\left(\eta \zeta \chi_{\psi^{*}}(\tau \wedge T)+(1-\eta) \zeta \chi_{\psi}(\tau \wedge T)\right)\right] \\
& +\zeta\left(W_{0}-n p_{0}+\mathbb{E}\left[\int_{0}^{\tau \wedge T} y_{t}\left(\eta \chi_{\psi^{*}}(t)+(1-\eta) \chi_{\psi}(t)\right) \mathrm{d} t+n\left(\eta \chi_{\psi^{*}}(\tau \wedge T)+(1-\eta) \chi_{\psi}(\tau \wedge T)\right) X(\tau \wedge T)\right]\right), \eta \in[0,1] .
\end{aligned}
$$

Then, by using the definition of $I_{0}^{(1)}$ and $J$, we have

$$
\begin{aligned}
G^{\prime}(\eta)= & -\zeta \mathbb{E}\left[\int_{0}^{\tau \wedge T} I_{0}^{(1)}\left(\eta \zeta \chi_{\psi^{*}}(t)+(1-\eta) \zeta \chi_{\psi}(t), t\right) \times\left(\chi_{\psi^{*}}(t)-\chi_{\psi}(t)\right) \mathrm{d} t\right. \\
& +J\left(\eta \zeta \chi_{\psi^{*}}(\tau \wedge T)+(1-\eta) \zeta \chi_{\psi}(\tau \wedge T)\right) \times\left(\chi_{\psi^{*}}(\tau \wedge T)-\chi_{\psi}(\tau \wedge T)\right) \\
& \left.-\int_{0}^{\tau \wedge T} y_{t}\left(\chi_{\psi^{*}}(t)-\chi_{\psi}(t)\right) \mathrm{d} t-n X(\tau \wedge T)\left(\chi_{\psi^{*}}(\tau \wedge T)-\chi_{\psi}(\tau \wedge T)\right)\right]
\end{aligned}
$$

and

$$
\begin{aligned}
G^{\prime \prime}(\eta)= & -\zeta^{2} \mathbb{E}\left[\int_{0}^{\tau \wedge T} I_{0}^{(1)}\left(\eta \zeta \chi_{\psi^{*}}(t)+(1-\eta) \zeta \chi_{\psi}(t), t\right) \times\left(\chi_{\psi^{*}}(t)-\chi_{\psi}(t)\right)^{2} \mathrm{~d} t\right. \\
& \left.+J^{\prime}\left(\eta \zeta \chi_{\psi^{*}}(\tau \wedge T)+(1-\eta) \zeta \chi_{\psi}(\tau \wedge T)\right) \times\left(\chi_{\psi^{*}}(\tau \wedge T)-\chi_{\psi}(\tau \wedge T)\right)^{2}\right] \geq 0 .
\end{aligned}
$$

Thus $G(\eta)$ is a convex function of $\eta$. If $\psi^{*}$ is a solution of the original problem, then $G(\eta)$ achieves its minimum at $\eta=1$. This is possible if and only if $G^{\prime}(1) \leq 0$. Explicitly, $\psi^{*}$ is a solution if and only if, for every other $\psi$ that satisfies 


$$
\begin{aligned}
& \mathbb{E}\left[\int_{0}^{\tau \wedge T} I_{0}^{(1)}\left(\zeta \chi_{\psi}(t)\right) \chi_{\psi}(t) \mathrm{d} t+J\left(\zeta \chi_{\psi}(\tau \wedge T)\right) \chi_{\psi}(\tau \wedge T)\right] \\
& \leq \mathbb{E}\left[\int_{0}^{\tau \wedge T} y(t) \chi_{\psi}(t) \mathrm{d} t+n \chi_{\psi}(\tau \wedge T) X(\tau \wedge T)\right]+W_{0}-n p_{0},
\end{aligned}
$$

we have

$$
\begin{aligned}
& \mathbb{E}\left[\int_{0}^{\tau \wedge T} I_{0}^{(1)}\left(\zeta \chi_{\psi^{*}}(t), t\right) \chi_{\psi^{*}}(t) \mathrm{d} t+J\left(\zeta \chi_{\psi^{*}}(\tau \wedge T)\right) \chi_{\psi^{*}}(\tau \wedge T)-\int_{0}^{\tau \wedge T} y(t) \chi_{\psi^{*}}(t) \mathrm{d} t-n \chi_{\psi^{*}}(\tau \wedge T) X(\tau \wedge T)\right] \\
& \geq \mathbb{E}\left[\int_{0}^{\tau \wedge T} I_{0}^{(1)}\left(\zeta \chi_{\psi^{*}}(t), t\right) \chi_{\psi}(t) \mathrm{d} t+J\left(\zeta \chi_{\psi^{*}}(\tau \wedge T)\right) \chi_{\psi}(\tau \wedge T)-\int_{0}^{\tau \wedge T} y(t) \chi_{\psi}(t) \mathrm{d} t-n \chi_{\psi}(\tau \wedge T) X(\tau \wedge T)\right] .
\end{aligned}
$$

We first note that, from Fubini's Theorem,

$$
\mathbb{E}\left[\int_{0}^{\tau \wedge T} I_{0}^{(1)}\left(\zeta \chi_{\psi}(t), t\right) \chi_{\psi}(t) \mathrm{d} t\right]=\int_{0}^{T} \mathbb{E}\left[I_{0}^{(1)}\left(\zeta \chi_{\psi}(t)\right) \chi_{\psi}(t) \times\left(1_{\{\tau>t\}}+1_{\{\tau \in(t-, t]\}}\right)\right] \mathrm{d} t .
$$

Since $I_{0}^{(1)}(x, t)$ is convex w.r.t. $\quad x \in \mathbb{R}^{+}$,

$$
I_{0}^{(1)}\left(\zeta \chi_{\psi}(t), t\right) \geq I_{0}^{\prime(1)}\left(\zeta \chi_{\lambda}(t), t\right) \zeta\left(\chi_{\psi}(t)-\chi_{\lambda}(t)\right)+I_{0}^{(1)}\left(\zeta \chi_{\lambda}(t), t\right) .
$$

Using the such facts that $\chi_{\psi}(t)=\chi_{\lambda}(t) \chi_{\psi}^{N}(t)$ and that $\chi_{\lambda}(t)$ is uncorrelated to both $\chi_{\psi}^{N}(t)$ and $\tau$, we have

$$
\begin{aligned}
& \mathbb{E}\left[I_{0}^{\prime(1)}\left(\zeta \chi_{\lambda}(t), t\right)\left(\chi_{\psi}(t)-\chi_{\lambda}(t)\right) \chi_{\psi}(t) \times\left(1_{\{\tau>t\}}+1_{\{\tau \in(t-t]\})}\right)\right] \\
& \left.=\mathbb{E}\left[I_{0}^{(1)}\left(\chi_{\lambda}(t), t\right)\right) \chi_{\lambda}(t)^{2}\right] \times \mathbb{E}\left[\left(\chi_{\psi}^{N}(t)^{2}-\chi_{\psi}^{N}(t)\right)\left(1_{\{\tau>t\}}+1_{\{\tau \in(t-, t]\}}\right)\right] .
\end{aligned}
$$

Hence, if the absolute value of Equation (48) is sufficiently small, from Equation (47),

$$
\mathbb{E}\left[I_{0}^{(1)}\left(\chi_{\psi}(t), t\right) \chi_{\psi}(t)\left(1_{\{\tau>t\}}+1_{\{\tau \in(t-, t]\}}\right)\right] \geq \mathbb{E}\left[I_{0}^{(1)}\left(\zeta \chi_{\lambda}(t), t\right) \chi_{\psi}(t)\left(1_{\{\tau>t\}}+1_{\{\tau \in(t-, t]\}}\right)\right] .
$$

From Equations (46) and (49), we obtain an inequality;

$$
\mathbb{E}\left[\int_{0}^{\tau \wedge T} I_{0}^{(1)}\left(\zeta \chi_{\psi}(t), t\right) \chi_{\psi}(t) \mathrm{d} t\right] \geq \mathbb{E}\left[\int_{0}^{\tau \wedge T} I_{0}^{(1)}\left(\zeta \chi_{\lambda}(t), t\right) \chi_{\psi}(t) \mathrm{d} t\right] .
$$

Quite similar arguments lead to an inequality;

$$
\mathbb{E}\left[J\left(\zeta \chi_{\psi}(\tau \wedge T), t\right) \chi_{\psi}(\tau \wedge T)\right] \geq \mathbb{E}\left[J\left(\zeta \chi_{\lambda}(\tau \wedge T), t\right) \chi_{\psi}(\tau \wedge T)\right] .
$$

Therefore, from Equations (50) and (51), we have

$$
\begin{aligned}
& \mathbb{E}\left[\int_{0}^{\tau \wedge T} I_{0}^{(1)}\left(\zeta \chi_{\psi}(t), t\right) \chi_{\psi}(t) \mathrm{d} t+J\left(\zeta \chi_{\psi}(\tau \wedge T)\right) \chi_{\psi}(\tau \wedge T)-\int_{0}^{\tau \wedge T} y(t) \chi_{\psi}(t) \mathrm{d} t-n \chi_{\psi}(\tau \wedge T) X(\tau \wedge T)\right] \\
& \geq \mathbb{E}\left[\int_{0}^{\tau \wedge T} I_{0}^{(1)}\left(\zeta \chi_{\lambda}(t), t\right) \chi_{\psi}(t) \mathrm{d} t+J\left(\zeta \chi_{\lambda}(\tau \wedge T)\right) \chi_{\psi}(\tau \wedge T)-\int_{0}^{\tau \wedge T} y(t) \chi_{\psi}(t) \mathrm{d} t-n \chi_{\psi}(\tau \wedge T) X(\tau \wedge T)\right] .
\end{aligned}
$$

So that, if we choose a $\zeta$ so that it satisfies

$\mathbb{E}\left[\int_{0}^{\tau \wedge T} I_{0}^{(1)}\left(\zeta \chi_{\lambda}(t), t\right) \chi_{\lambda}(t) \mathrm{d} t+J\left(\zeta \chi_{\lambda}(\tau \wedge T)\right) \chi_{\lambda}(\tau \wedge T)-\int_{0}^{\tau \wedge T} y(t) \chi_{\lambda}(t) \mathrm{d} t-n \chi_{\lambda}(\tau \wedge T) X(\tau \wedge T)\right]=W_{0}-n p_{0}$,

then we have

$$
\begin{aligned}
& \mathbb{E}\left[\int_{0}^{\tau \wedge T} I_{0}^{(1)}\left(\zeta \chi_{\lambda}(t), t\right) \chi_{\lambda}(t) \mathrm{d} t+J\left(\zeta \chi_{\lambda}(\tau \wedge T)\right) \chi_{\lambda}(\tau \wedge T)-\int_{0}^{\tau \wedge T} y(t) \chi_{\lambda}(t) \mathrm{d} t-n \chi_{\lambda}(\tau \wedge T) X(\tau \wedge T)\right] \\
& \geq \mathbb{E}\left[\int_{0}^{\tau \wedge T} I_{0}^{(1)}\left(\zeta \chi_{\lambda}(t), t\right) \chi_{\psi}(t) \mathrm{d} t+J\left(\zeta \chi_{\lambda}(\tau \wedge T)\right) \chi_{\psi}(\tau \wedge T)-\int_{0}^{\tau \wedge T} y(t) \chi_{\psi}(t) \mathrm{d} t-n \chi_{\psi}(\tau \wedge T) X(\tau \wedge T)\right]
\end{aligned}
$$


holds by Equation (44). If we compare Equation (54) with Equation (45), $\lambda$ must be $\psi^{*}$ and this argument completes the proof. Finally, we note that there exists a unique $\zeta$ satisfying Equation (53) since we have

$$
\begin{gathered}
\lim _{x \rightarrow \infty} U_{i}^{\prime}(x)=0, \lim _{x \rightarrow 0+} U_{i}^{\prime}(x)=\infty, U_{i}^{\prime \prime}(x)<0, \\
i=1,2, x>0,
\end{gathered}
$$

and

$$
V^{\prime}(x)=\mathcal{Z}(x), V_{\tau \wedge T}^{\prime \prime}(x)=\mathcal{Z}^{\prime}(x)<0, x>0,
$$

from Assumption 2 and the definition of $U_{i}, i=1,2$, and $V$.

\section{Proof of Proposition 3:}

For each $n \in \mathbb{R}_{+}$, let $\hat{V}_{0}(n)$ be defined by

$$
\hat{V}_{0}(n)=\min _{\zeta^{(n)} \in \mathbb{R}_{++}} V_{0}\left(\zeta^{(n)}, \lambda\right) .
$$

Then, we can readily show that

$$
\begin{aligned}
\hat{V}_{0}(n)= & \mathbb{E}\left[\int_{0}^{\tau \wedge T} \tilde{u}_{0}^{(1)}\left(\hat{\zeta}^{(n)} \chi_{\lambda}(t), t\right) \mathrm{d} t+\tilde{V}_{\tau \wedge T}\left(\hat{\zeta}^{(n)} \chi_{\lambda}(\tau \wedge T)\right)\right. \\
& \left.+\hat{\zeta}^{(n)}\left(W(0)-n p_{0}+\int_{0}^{\tau \wedge T} \chi_{\lambda}(t) y(t) \mathrm{d} t+\chi_{\lambda}(\tau \wedge T) n X(\tau \wedge T)\right)\right] .
\end{aligned}
$$

Here, noting that, from Equation (24),

$$
\begin{aligned}
& \mathbb{E}\left[\int_{0}^{\tau \wedge T} \chi_{\lambda}(t) I_{0}^{(1)}\left(\hat{\zeta}^{(n)} \chi_{\lambda}(t), t\right) \mathrm{d} t+\chi_{\lambda}(\tau \wedge T) \hat{W}(\tau \wedge T)\right] \\
& =W_{0}-n p_{0}+\mathbb{E}\left[\int_{0}^{\tau \wedge T} \chi_{\lambda}(t) y(t) \mathrm{d} t+n \chi_{\lambda}(\tau \wedge T) X(\tau \wedge T)\right]
\end{aligned}
$$

holds, we obtain

$$
\begin{aligned}
\hat{V}_{0}^{\prime}(n) & :=\frac{\mathrm{d} \hat{V}_{0}(n)}{\mathrm{d} n} \\
& =\hat{\zeta}^{(n)}\left(\mathbb{E}\left(\chi_{\lambda}(\tau \wedge T) X(\tau \wedge T)\right)-p_{0}\right) .
\end{aligned}
$$

Since $\hat{\zeta}^{(n)}>0$, Equation (56) immediately leads to the result.

\section{Proof of Proposition 4:}

First, we define some notations as follows.

$$
\begin{gathered}
P_{t}(\tau \in \mathrm{d} s)=\lambda(s) \exp \left\{-\int_{t}^{s} \lambda(v) \mathrm{d} v\right\} \mathrm{d} s, \\
P_{t}(\tau>u)=\exp \left\{-\int_{t}^{u} \lambda(v) \mathrm{d} v\right\}, \\
\mathcal{E}_{t}(u, z):=\exp \left\{\alpha_{t}(u)+\gamma_{t}(u) z\right\}, \\
\alpha_{t}(u):=-\int_{t}^{u} r(s) \mathrm{d} s-\frac{1}{2} \int_{t}^{u} \xi(s)^{2} \mathrm{~d} s,
\end{gathered}
$$

$$
\gamma_{t}(u):=\left(\int_{t}^{u} \xi(s)^{2} \mathrm{~d} s\right)^{\frac{1}{2}} .
$$

We note that if $Z \sim N(0,1)$, that is, if $Z$ is a random variable that follows the standard normal distribution, a process $\left\{\mathcal{E}_{t}(u, Z) ; u \in[t, T]\right\}$ has an identical distribution with that of the state price density process $\{\chi(u) ; u \in[t, T]\}$ conditioned by $\mathcal{F}_{t}$.

Now, we consider the value of the household's wealth $\hat{W}_{t}$ at time $t$. If $\tau \geq t$, then

$$
\hat{W}(t)= \begin{cases}\hat{W}(t-)+n X(t) & \text { if } \tau=t, \\ \hat{W}(t-) & \text { if } \tau>t .\end{cases}
$$

On the other, considering total net value at time $t$ of optimal future consumption of the household, if $\tau=t$,

$$
\hat{W}(t)=J\left(\zeta^{*} \chi(t)\right),
$$

otherwise, if $\tau>t$,

$$
\hat{W}(t)=(T C)_{t}-(T Y)_{t}-(T X)_{t},
$$

where

$$
\begin{gathered}
(T C)_{t}:=\mathbb{E}_{t}\left[\int_{t}^{\tau \wedge T} \mathcal{E}_{t}(u, Z) \times I_{0}^{(1)}\left(\zeta^{*} \chi(t) \mathcal{E}_{t}(u, Z), u\right) \mathrm{d} u+\mathcal{E}_{t}(\tau \wedge T, Z) J\left(\zeta^{*} \chi(t) \mathcal{E}_{t}(\tau \wedge T, Z)\right)\right], \\
(T Y)_{t}:=\mathbb{E}_{t}\left[\int_{t}^{T} \mathcal{E}_{t}(u, Z) y(u) 1_{\{\tau>u\}} \mathrm{d} u\right]=\int_{t}^{T} y(u) \exp \left\{-\int_{t}^{u}(r(s)+\lambda(s)) \mathrm{d} s\right\} \mathrm{d} u,
\end{gathered}
$$

and

$$
\begin{aligned}
& (T X)_{t}:=n \mathbb{E}_{t}\left[\mathcal{E}_{t}(\tau \wedge T, Z) X(\tau \wedge T)\right]=n x \mathbb{E}_{t}\left[\int_{t}^{T} \mathcal{E}_{t}(s, Z)(1+H(s)) P_{t}(\tau \in \mathrm{d} s)+\mathcal{E}_{t}(T, Z) P_{t}(\tau>T)\right] \\
& =n x\left(\int_{t}^{T}(1+H(s)) \times \lambda(s) \exp \left\{-\int_{t}^{s}(r(u)+\lambda(u)) \mathrm{d} u\right\} \mathrm{d} s+\exp \left\{-\int_{t}^{T}(r(s)+\lambda(s)) \mathrm{d} s\right\}\right) .
\end{aligned}
$$


Here, we note that $(T Y)_{t}$ denotes time $t$ value of the household's income to be gained in the future and that $(T X)_{t}$ denotes time $t$ value of the money from insurance paid if $\tau$ occurs before time $T$.

Therefore, from Equation (61) and Equation (63), if $\tau \geq t$,

$$
\begin{aligned}
\mathrm{d} \hat{W}(t) & =\mathrm{d} \hat{W}(t-)+n X(t) \mathrm{d} N(t) \\
& =\mathrm{d}(T C)_{t^{-}}-\mathrm{d}(T Y)_{t^{-}}-\mathrm{d}(T X)_{t^{-}}+n X(t) \mathrm{d} N(t) .
\end{aligned}
$$

Next, we derive differential, $\mathrm{d}(T C)_{t-}, \mathrm{d}(T Y)_{t-}$ and $\mathrm{d}(T X)_{t-}$, explicitly. Since

$$
\begin{aligned}
(T C)_{t-}= & \int_{t}^{T} \int_{-\infty}^{\infty}\left(\int_{t}^{s} \mathcal{E}_{t}(u, z) I_{0}^{(1)}\left(\zeta^{*} \chi(t) \mathcal{E}_{t}(u, z), u\right) \mathrm{d} u+\mathcal{E}_{t}(s, z) J\left(\zeta^{*} \chi(t) \mathcal{E}_{t}(s, z)\right)\right) \mathrm{d} \Phi(z) P_{t}(\tau \in \mathrm{d} s) \\
& +\int_{-\infty}^{\infty}\left(\int_{t}^{T} \mathcal{E}_{t}(u, z) I_{0}^{(1)}\left(\zeta^{*} \chi(t) \mathcal{E}_{t}(u, z), u\right) \mathrm{d} u+\mathcal{E}_{t}(T, z) J\left(\zeta^{*} \chi(t) \mathcal{E}_{t}(T, z)\right)\right) \mathrm{d} \Phi(z) P_{t}(\tau>T),
\end{aligned}
$$

where $\Phi(z)$ is the c.d.f. of the standard normal distribution. Note that the last equation holds by Fubini's theorem. A straightforward but long algebra leads to

$$
\begin{aligned}
\mathrm{d}(T C)_{t-}= & -I_{0}^{(1)}\left(\zeta^{*} \chi(t), t\right) \mathrm{d} t+(T C)_{t^{-}} r(t) \mathrm{d} t-\zeta^{*} \chi(t) \mathbb{E}_{t-}\left[\int_{t}^{\tau \wedge T} \mathcal{E}_{t}(u, Z)^{2} \times I_{0}^{(1)}\left(\zeta^{*} \chi(t) \mathcal{E}_{t}(u, Z), u\right) \mathrm{d} u\right. \\
& \left.+\mathcal{E}_{t}(\tau \wedge T, Z)^{2} J^{\prime}\left(\zeta^{*} \chi(t) \mathcal{E}_{t}(\tau \wedge T, Z)\right)\right] \times\left(\xi(t)^{2} \mathrm{~d} t+\xi(t) \mathrm{d} B(t)\right)-\left(J\left(\zeta^{*} \chi(t)\right)-(T C)_{t-}\right) \lambda(t) \mathrm{d} t .
\end{aligned}
$$

Similarly, from Equations (65) and (66), we can readily confirm that

$$
\begin{aligned}
\mathrm{d}(T X)_{t-} & =-n X(1+H(t)) \lambda(t) \mathrm{d} t+(T X)_{t-}(r(t)+\lambda(t)) \mathrm{d} t \\
& =(T X)_{t_{-}} r(t) \mathrm{d} t-\left(n X(t)-(T X)_{t^{-}}\right) \lambda(t) \mathrm{d} t
\end{aligned}
$$

and

$$
\mathrm{d}(T Y)_{t-}=(T Y)_{t-}(r(t)+\lambda(t)) \mathrm{d} t-y(t) \mathrm{d} t
$$

hold. Therefore, from Equations (67), (69)-(71), we obtain

$$
\begin{aligned}
\mathrm{d} \hat{W}(t)= & \mathrm{d}(T C)_{t-}-\mathrm{d}(T X)_{t-}-\mathrm{d}(T Y)_{t-}+n X(t) \mathrm{d} N(t) \\
= & y(t) d t-\hat{c}(t) \mathrm{d} t+\hat{W}(t-) r(t) \mathrm{d} t+\hat{\pi}(t)((\mu(t)-r(t)) \mathrm{d} t+\sigma(t) \mathrm{d} B(t)) \\
& -\left[J\left(\zeta^{*} \chi(t)\right)-n X_{t}-\hat{W}(t-)\right] \lambda(t)+n X(t) \mathrm{d} N(t) .
\end{aligned}
$$

From Equation (61), since

$J\left(\zeta^{*} \chi(t)\right)=\hat{W}(t-)+n X(t)$, we can conclude that the proposition holds. 